\title{
Bonding Effects in Nitrogen Doped Graphene and Hexagonal Boron Nitride
}

\author{
J. C. Meyer,* S. Kurasch,* H.-J. Park, ${ }^{* *}$ V. Skakalova, ${ }^{* *}$ D. Künzel, ${ }^{* * * A . ~ G r o ß, ~}{ }^{* * *}$ A. Chuvilin, ${ }^{*}{ }^{1}$ S. \\ Roth,**** and U. Kaiser* \\ * Electron Microscopy of the Materials Sciences, University of Ulm, Germany \\ ** Max Planck Institute for Solid State Research, Stuttgart, Germany \\ *** Institute for Theoretical Chemistry, University of Ulm, Germany \\ **** School of Electrical Engineering, WCU Flexible Nanosystems, Korea University, Seoul, Korea
}

We report on the effects of chemical bonding on high-resolution transmission electron microscopy images of covalently bonded light elements $(\mathrm{B}, \mathrm{C}, \mathrm{N})$. We follow the procedure of Deng and Marks [1] (with some modifications) to calculate the accurate electrostatic potential of the bonded configuration using the WIEN2k DFT code [2]. The conventional TEM simulation, based on the independent atom model (IAM), is shown for comparison. We have studied single-layer hexagonal boron nitride and nitrogen-doped graphene membranes by calculation and experiment. Nitrogen doped graphene membranes are prepared by CVD methods [3] with the addition of ammonia into the reaction [4]. TEM imaging is carried out using an image-side Cs-corrected Titan $80-300$, operated at $80 \mathrm{kV}$. The spherical aberration is $20 \mu \mathrm{m}$ and a defocus of $\mathrm{f} 1=-90 \AA$ (Scherzer defocus) and $\mathrm{f} 2=-180 \AA$ is used.Image calculations for nitrogen doped graphene are based on a DFT relaxed atomic configuration. This configuration was obtained with the VASP DFT code [5], and agrees with previous works [6,7] in that the bond length changes after replacing $\mathrm{C}$ by $\mathrm{N}$ are less than $2 \mathrm{pm}$. According to conventional (IAM) TEM simulation, a nitrogen substitution in graphene would be invisible (a contrast of less than $0.1 \%$ ) for all these relaxed configurations. Image simulations based on the electrostatic potentials from the DFT calculation, however, predict a detectable signal. Our charged defect produces predominantly low -er spatial frequency information in the projected potentials. This is mostly cut off by the CTF in the Scherzer-focus image of the Cs-corrected microscope (f1, top row in Fig. 1), but visible at larger defo -cus. A further analysis of the projected potentials and electron distribution shows that the chemical shift here is not localized on the nitrogen substitution, but is actually spread onto the neighbouring car -bons. We note that a physical phase plate (not present in the current experiment) would be highly useful to study this non-local bonding effect to include all frequencies in one image. The third row in Fig. 1 shows an example experimental image from nitrogen doped single layer graphene membranes recorded at $\mathrm{f} 2$ (a drift-compensated average of 34 individual exposures). Two defects in excellent agreement with expectations (a dark contrast of ca. $0.5 \%$ ) are visible in this image. In in-focus (f1) images of the same noise level of the same defects, the substitutions could not be detected above the noise (in agreement with the calculation).

We also briefly consider the IAM and DFT calculation results for single-layer hexagonal boron nitride (h BN). Fig. 2 shows the projected electrostatic potentials for IAM and DFT, withdifferent resolutionlimiting apertures. The IAM simulation predicts a small but significant contrast difference for the two

${ }^{1}$ Current address: CIC nanoGUNE Consolider, San Sebastian, and Ikerbasque, Basque Foundation for Science, Bilbao, Spain 
elements, already with only $2.16 \AA$ (1-100 reflection) resolution. The DFT based simulation, on the other hand, predicts a practically symmetric image in this case. Although the difference between the IAM and DFT result is small, it has important implications: The identification of the $\mathrm{B}$ and $\mathrm{N}$ is sites not possible at only $2.16 \AA$ resolution. For the experimental case, the separation of intrinsic contrast (i.e., that of the sample) and effects of optical aberrations (from the microscope) is lengthy and can not be treated in this abstract.

In conclusion, the bonding effects enable the detection of the isolated nitrogen dopant in graphene $(\mathrm{dZ}$ $=1)$, but suppress the contrast difference of $\mathrm{B}$ and $\mathrm{N}$ in $\mathrm{h}-\mathrm{BN}(\mathrm{dZ}=2)$ at the lower resolution. Clearly, bonding effects should be taken into account for the analysis of images from these materials.

We gratefully acknowledge financial support by the Deutsche Forschungsgemeinschaft (DFG) within the SALVE project and SFB 569 and by the Landesstiftung Baden-Württemberg.

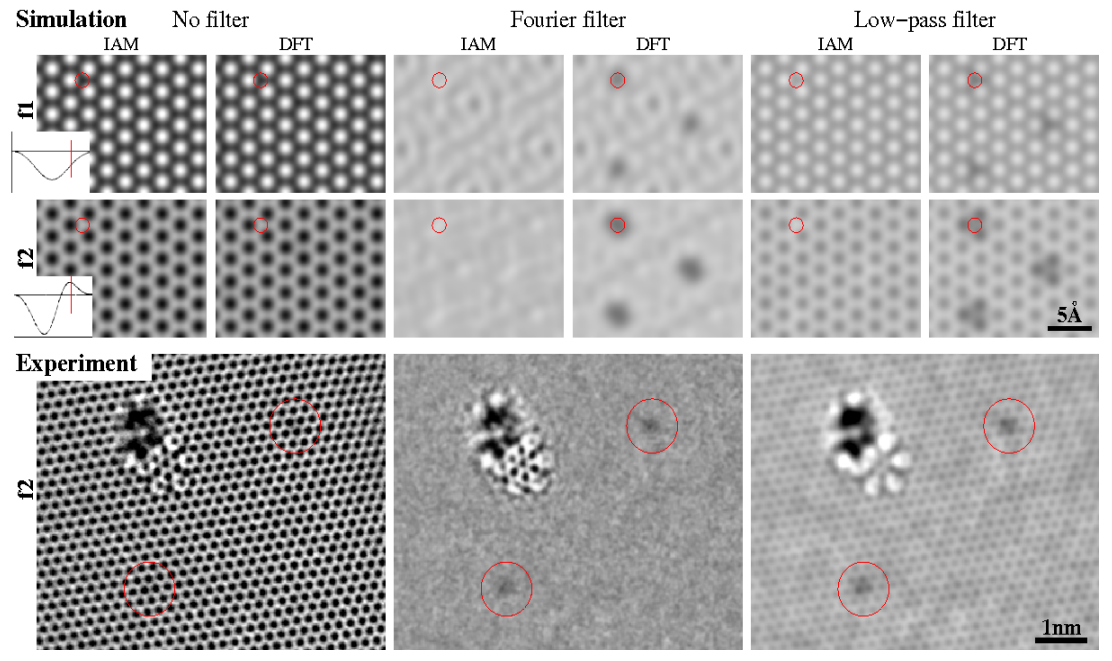

Figure 1: Simulation and experiment for nitrogen doped graphene. The nitrogen substitutions in graphene are detectable only due to a bonding effect. They produce a mostly lowerfrequency contrast that is best detected at a larger defocus (f2 $=-18 \mathrm{~nm}$ ). In the simulated images one of the three dopant atoms is marked. The defect is visible after removing the periodic graphene lattice (center) or reducing the lattice contrast by a lowpass filter (gauss shaped).

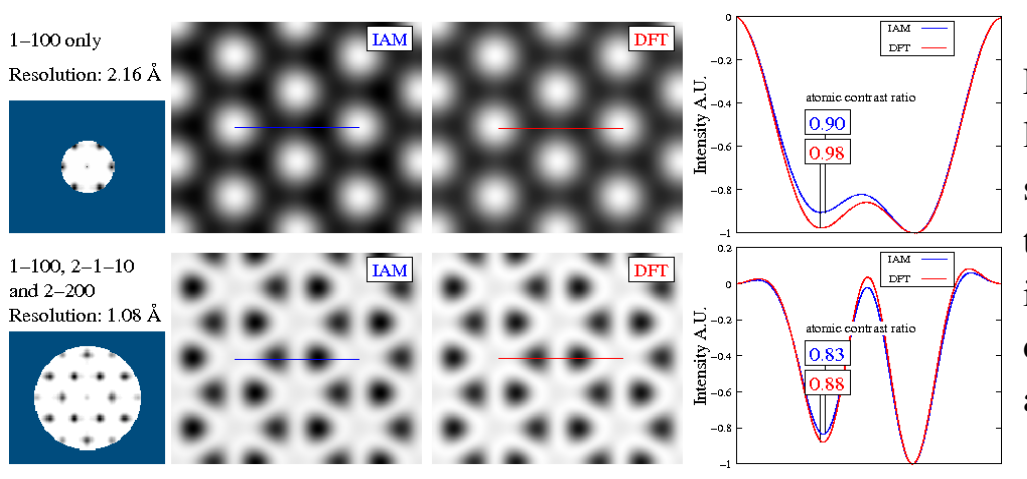

Figure 2: Projected potentials of hBN monolayers at different resolution (black corresponds to high projected potential, with intensity minima at the atom positions). The ionicity of the material cancels the contrast difference at a spatial resolution of $2.16 \AA$ according to the DFT result.

[1] B. Deng and L. D. Marks. Acta Cryst. A, 62 (2006) 208. [2] P. Blaha, K. Schwarz, G. K. H. Madsen, D. Kvasnicka, and J. Luitz. WIEN2K: An Augmented Plane Wave and Local Orbitals Program for Calculating Crystal Properties. Vienna University of Technology, Vienna, Austria (2001). [3] K. S. Kim et al., Nature, 457 (2009) 706. [4] D. Wei et al., Nano Lett., 9 (2009) 1752.

[5] G. Kresse and J. Furthmüller. Phys. Rev. B, 54:11169, 1996. [6] Z. H. Zhu et al., J. Phys. Chem. B, 109 (2005) 16744. [7] S. H. Lim et al., Phys. Rev. B, 76 (2007) 195406. 